\title{
Modeling Breast Cancer Intertumor and Intratumor Heterogeneity Using Xenografts
}

\author{
Alejandra Bruna, ${ }^{1}$ Oscar M. Rueda,${ }^{1}$ and Carlos Caldas ${ }^{1,2}$ \\ ${ }^{1}$ Department of Oncology and Cancer Research UK Cambridge Institute, Li Ka Shing Centre, University \\ of Cambridge, Cambridge CB2 ORE, United Kingdom \\ ${ }^{2}$ Breast Cancer Programme, Cambridge Cancer Centre, Cambridge CB2 2QQ, United Kingdom \\ Correspondence: carlos.caldas@cruk.cam.ac.uk
}

\begin{abstract}
Breast cancer is a heterogeneous disease that can be stratified in at least 10 different subtypes. We present here a platform for derivation of preclinical models based on patient-derived tumor xenografts (PDTXs) that represent these subgroups. These models preserve the transcriptome, methylome, copy-number, and mutational landscape features of the tumor of origin through different passaging. Furthermore, the intratumoral composition of these models is formed by communities of clones very similar to the ones present in the originating tumor. Finally, we show that short-term cultures of cells from these models (PDTX-derived tumor cells, PDTCs) also preserve the molecular features of the tumor and can be used for high-throughput drug testing of single compounds or combinations, with high reproducibility and clinical predictive power.
\end{abstract}

Breast cancer is a heterogeneous disease. Consequently, precise breast cancer stratification is a crucial step toward more efficacious treatment. For many years patients have been stratified based on clinical-pathological features including staging, tumor grade, and the estrogen receptor (ER), progesterone receptor (PR), and Her2 status. Molecular stratification based on gene expression profiling defined the five intrinsic subtypes of breast cancer: basal-like, Her2, luminal A, luminal B, and normallike (Perou et al. 2000).

However, these classifications are not optimal. Clinical and histological stratification still fails to assign patients to the right treatment; in particular within the largest subset of patients, which are $\mathrm{ER}^{+} / \mathrm{Her} 2^{-}$, where hormone therapy, with Tamoxifen or aromatase inhibitors, frequently fails, and where use of chemotherapy is fraught with poor prediction of benefit. This problem persists even using the gene expression-based intrinsic subtype classification, where luminal A and luminal B are very broad groups that only differ in proliferation status. We therefore hypothesized that a taxonomy of breast cancer based on genomic driver events would be an improvement, which would ultimately lead to better treatment decisions. The METABRIC (Molecular Taxonomy of Breast Cancer International Consortium) project (Curtis et al. 2012) used copy-number and expression profiling of 997 primary breast cancer samples to identify the top 1000 candidate driver genes based on their expression being driven in cis by copy-number aberrations. Using these $1000 \mathrm{cis}$-driven genes, enriching for oncogenes and tumor-suppressor genes, we showed that breast cancers could be divided into 10 integrative clusters (IntClusts). This molecular stratification was validated in a further 983 samples from the same cohort (Curtis et al. 2012) and subsequently in several external data sets (Ali et al. 2014). Some of the IntClusts were characterized by a very clear copy-number driver event, like the $C C N D 1 / P A K 1$ amplification in IntClust 2, the HER2 amplification in IntClust 5, or the MYC amplicon in IntClust9, but in some other groups drivers were more elusive. To gain insight into different molecular events, we also performed targeted sequencing of 173 genes (Ali et al. 2014) that are relevant in breast cancer, finding that 40 of them are drivers (mut-driver genes), with different frequencies in each IntClust. This new layer of molecular data contributed to refining the molecular landscape of breast cancer. Significantly, some of these mutation drivers seem to have IntClust specific prognostic value. For example, $P I K 3 C A$ is associated with worse survival in only three $\mathrm{ER}^{+} / \mathrm{Her} 2^{-}$subgroups. We also quantified the level of intratumoral heterogeneity in individual tumors, revealing its distribution is distinctly different between IntClusts. Importantly we also showed that intratumoral heterogeneity is a biomarker of diseasespecific survival.

However, all of these important biological associations that we can infer with a well-annotated and well-characterized cohort like METABRIC need a set of representative preclinical models in order to be validated. With this aim in mind, we developed a classifier to assign existing breast cancer cell lines into one of the IntClusts (Curtis et al. 2012). We observed the fit was poor because the genomic profiles of the cell lines did not reflect well those found in breast cancer samples in the clinic. This is a consequence of the nature of cell lines, which not only fail to capture the intratumor and intertumor heterogeneity seen in the clinic but also have other limitations probably reflecting adaptation to long-term culture in plastic. These drawbacks are thought to have strongly impacted

(C) 2016 Bruna et al. This article is distributed under the terms of the Creative Commons Attribution-NonCommercial License, which permits reuse and redistribution, except for commercial purposes, provided that the original author and source are credited. 
cancer drug development, which has suffered from high attrition rates, mainly because of lack of efficacy in the clinic (Harrison 2016).

\section{DEVELOPING A COLLECTION OF PATIENT- DERIVED TUMOR XENOGRAFTS}

Patient-derived tumor xenografts (PDTXs) have emerged as tools with the potential to improve cancer drug development (Tentler et al. 2012; Hidalgo et al. 2014; Aparicio et al. 2015; Gao et al. 2015). We therefore set out to develop PDTXs as a better model to study drug sensitivity but also to reflect tumor evolution under the selective pressure produced by treatment, enabling the study of mechanisms of sensitivity and resistance (Fig. 1). We have now optimized a framework to efficiently engraft breast cancer samples from the hospital into immunocompromised mice (NSG). With time we have substantially improved this platform to (1) ensure rapid and efficient implantation of new breast cancer samples, (2) expand the successful engrafted samples through serial transplants for biobanking purposes, and (3) provide these models as a research tool. The breast cancer PDTX biobank is now one of the largest in the academic setting and is composed by almost equal number of $\mathrm{ER}^{+}$ and $\mathrm{ER}^{-}$samples, most of which are from breast tumor primaries.

We have now robustly shown that the PDTXs represent the breast cancer patient population and preserve most of the features of the originating tumor sample. This has included full characterization at the transcriptome, methylome, copy-number, and mutational landscape levels, demonstrating similarity with the originating human tumor material and its preservation through several passages in the mouse.

We soon realized the downstream analysis of sequencing data was complicated by the nature of PDTX samples, which are unknown mixtures of mouse stromal and hu-

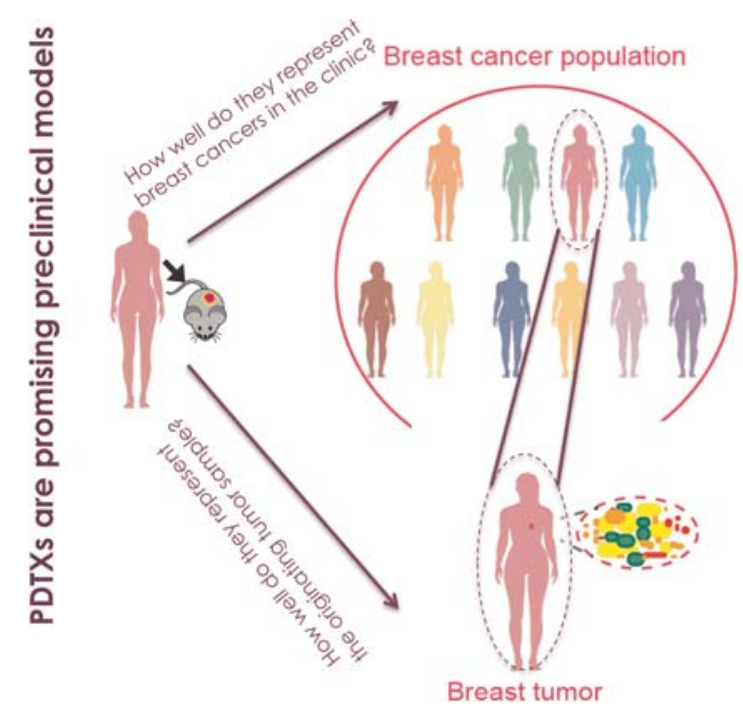

Figure 1. Modeling breast cancer intertumor and intratumor heterogeneity. PDTXs, patient-derived tumor xenografts. man epithelial malignant cells. This prompted the development of a bioinformatics pipeline that allowed for the identification with $99.9 \%$ accuracy of mouse and human DNA sequences. Significantly, we have observed the proportion of mouse stromal cells in the PDTXs remains stable through serial passages. These data were confirmed by orthogonal methods such as fluorescence in situ hybridization (FISH) and immunohistochemistry (Bruna et al. 2016).

We used exome sequencing to define single-nucleotide variations (SNVs) and small insertions and deletions (indels), shallow whole-genome sequencing to obtain copy-number profiles, Illumina expression microarrays (and RNA-seq) to measure gene expression and infer pathway activation, and reduced representation bisulfite sequencing (RRBS) to characterize DNA methylation.

We classified PDTXs into the IntClusts using the method we previously described (Ali et al. 2014). The copynumber profiles of PDTXs classified into each IntClust are similar to those reported in primary tumors, contrasting to what we observed in cell lines (Curtis et al. 2012; Ali et al. 2014). The subtype distribution of engrafted PDTX samples in the biobank is biased toward $\mathrm{ER}^{-}$ and $\mathrm{ER}^{+}$tumors with worse prognosis (Curtis et al. 2012; Bruna et al. 2016), reflecting the favored engraftment of more aggressive breast cancer subtypes. Hence, the $\mathrm{ER}^{-}$PDTX samples in the biobank have a distribution of most frequently mutated genes similar to that found in $\mathrm{ER}^{-}$breast cancers from the The Cancer Genome Atlas (TCGA) cohort (Cancer Genome Atlas 2012). In contrast, frequencies of mutated genes in $\mathrm{ER}^{+}$PDTX samples mirrored those found in more aggressive $\mathrm{ER}^{+}$ subtypes (Eirew et al. 2015).

When comparing PDTX to their matched originating sample and to later passages, we observed that overall the molecular profiles are remarkably similar. The biggest drift occurs with engrafting, although it is model-specific, suggesting there is not a common mechanism underlying changes observed when implanting. It is hence more likely that patient- and tumor-specific determinants, in combination with growing in a new environment in the immunodeficient mouse host, affects the observed drifts. Significantly, all the molecular traits examined remained remarkably stable through serial passages in the mouse, even after more than 10 passages (Bruna et al. 2016).

We further investigated the intratumoral composition and architecture of PDTX samples and observed xenografts are communities of clones with varying degrees of complexity similar to those observed in the breast cancer clinical population, and these communities share most of the clones found in the matched originating cancer sample (Bruna et al. 2016; Pereira et al. 2016). Because cancer genomes constantly evolve under selective pressure, we investigated the extent of the human-to-mouse and mouse-to-mouse clonal dynamics using PyClone (Roth et al. 2014; Eirew et al. 2015), a method for estimating the proportion of cells in a tumor carrying each mutation and clustering them into subclones (groups of cells with the same genotype). We observed that most of the clones remained stable after engraftment and throughout passag- 


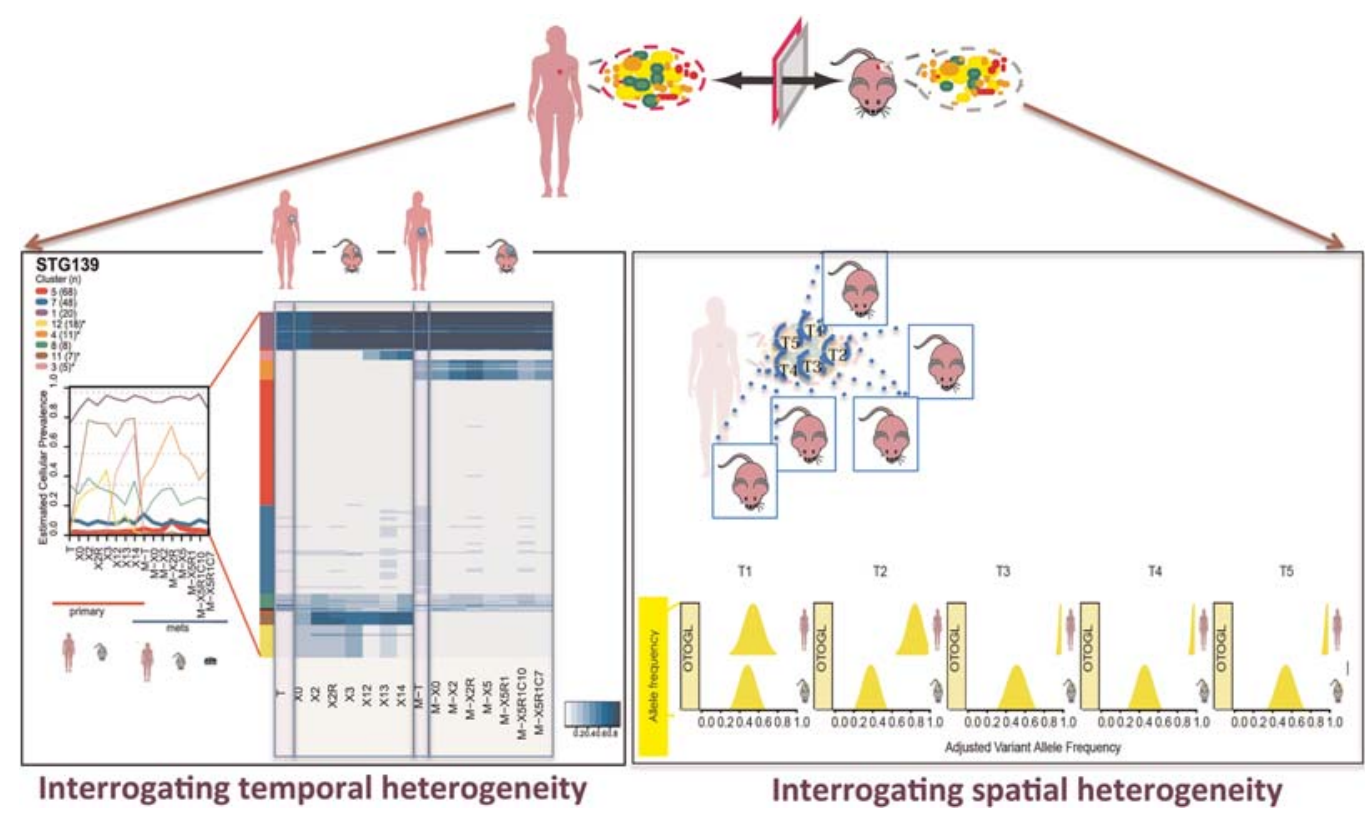

Figure 2. Patient-derived tumor xenografts (PDTXs) retain most of the molecular features of their originating tumor samples, including the intratumor heterogeneity, and remain very stable in the mouse. (Portions adapted from Bruna et al. 2016.)

ing, although in certain instances some subclones increased or decreased their prevalence or even appeared or disappeared. In fact, only $20 \%$ of the almost 200 clonal clusters detected changed significantly. Importantly, only four of these contained known breast cancer drivers. We further investigated the temporal and spatial intratumoral heterogeneity in a few examples. By comparing the clonal cluster composition and allele frequency distributions in matched primary, metastatic, and PDTX samples we observed both common and private clones. We further engrafted five spatially separated biopsies from a patient tumor sample and observed minor spatial intratumor heterogeneity. However, clonal dynamics were remarkably similar in independently engrafted mice regardless of their prevalence in the originating human tumor sample. Altogether, these results significantly added to previous data showing clones follow deterministic evolutionary trajectories (Fig. 2; Eirew et al. 2015).

The potential value of PDTXs gained further momentum with a study by Novartis showing xenografts captured the interpatient drug response variability (Gao et al. 2015). This suggested PDTXs would reach their full potential if their use would be possible for high-content studies. However, it is not feasible to do this in mice for ethical and economic reasons. This led us to develop a pipeline that would maintain and expand cancer samples in vivo in mice and then use the xenografts to generate single cells for short-term culture and high-throughput drug screening. These short-term cultures from PDTXs (PDTX-derived tumor cells or PDTCs) retain similar molecular features, including the intratumor heterogeneity, as in the originating PDTX sample (Fig. 3; Bruna et al. 2016). We then did a proof of concept screen, using the Welcome Trust Sanger Institute setup for high-throughput drug testing in cell lines (Garnett et al. 2012), in 22 PDTX models at different passages. We tested 102 compounds relevant to cancer, either as single agents or as drug:drug combinations in a $5 \times 5$ matrix of standard of care agents cisplatin and paclitaxel with six clinically relevant targeted compounds. We observed the technical and biological reproducibility was similar to that reported in established cell lines and organoids (Garnett et al. 2012; van de Wetering et al. 2015). To further verify the robustness of the data, we tested eight PDTC models using in parallel three end point DNA content- or ATP-dependent assays and observed highly correlated drug responses independently of the assay used. We also observed a very high correlation of responses in all models tested to pairs of compounds targeting the same pathway or sharing a similar mechanism of action. Furthermore, as an example of successful drug:drug combination approaches, we validated synergy between an HSP90 inhibitor and paclitaxel in Her $2^{+}$models (Bruna et al. 2016). These results support the idea that the PDTX/PDTC platform generates biologically robust data and shows its potential of becoming a resourceful intermediate in drug discovery. To further support the value of the PDTX/PDTC platform as an improved preclinical tool with clinical predictive power, we validated a set of ex vivo drug responses in vivo. Out of the 40 selected ex vivo drug responses, 33 were validated in vivo, even though different compounds with similar specificity had to be used in some cases.

In summary, we have redefined the molecular taxonomy of breast cancer using a genomic-driver-based stratification, and we have now generated a set of xenografts that model the intertumor and intratumor heterogeneity seen in the clinic. We have demonstrated these models can be used as a robust preclinical drug testing platform, and we will use them also for other perturbations to test functional heterogeneity. 
Generation of PDTCs

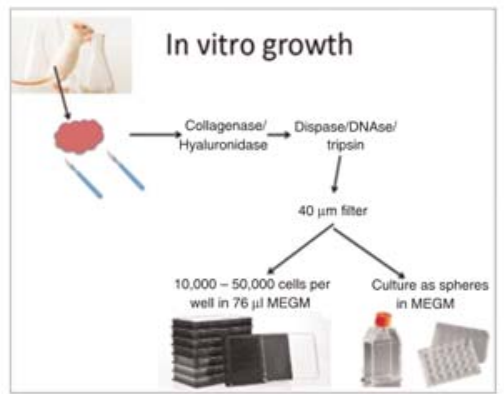

Preserve proportion of mouse and human cells

Percentage human DNA

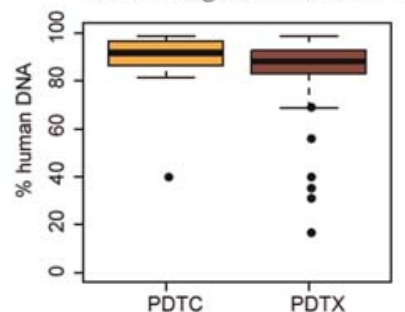

Preserve molecular features

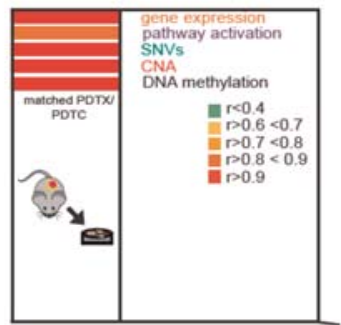

Preserve intratumor architecture

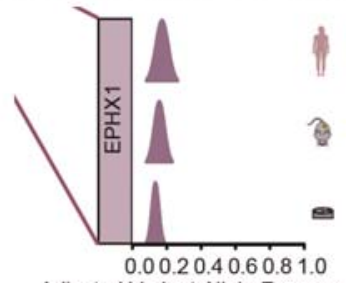

Figure 3. Generation of short-term cultures of PDTX-derived tumor cells (PDTCs) that preserve the proportion of mouse and human cells, the molecular features, and the intratumor heterogeneity of its originating sample. (Portions adapted from Bruna et al. 2016.)

\section{REFERENCES}

Ali HR, Rueda OM, Chin SF, Curtis C, Dunning MJ, Aparicio SA, Caldas C. 2014. Genome-driven integrated classification of breast cancer validated in over 7,500 samples. Genome Biol 15: 431 .

Aparicio S, Hidalgo M, Kung AL. 2015. Examining the utility of patient-derived xenograft mouse models. Nat Rev Cancer 15: 311-316.

Bruna A, Rueda OM, Greenwood W, Batra AS, Callari M, Batra RN, Pogrebniak K, Sandoval J, Cassidy JW, Tufegdzic-Vidakovic A, Sammut SJ, et al. 2016. A biobank of breast cancer explants with preserved intra-tumor heterogeneity to screen anticancer compounds. Cell 167: 260-274 e222.

Cancer Genome Atlas Network. 2012. Comprehensive molecular portraits of human breast tumours. Nature 490: 61-70.

Curtis C, Shah SP, Chin SF, Turashvili G, Rueda OM, Dunning MJ, Speed D, Lynch AG, Samarajiwa S, Yuan Y, et al. 2012. The genomic and transcriptomic architecture of 2,000 breast tumours reveals novel subgroups. Nature 486: 346352.

Eirew P, Steif A, Khattra J, Ha G, Yap D, Farahani H, Gelmon K, Chia S, Mar C, Wan A, et al. 2015. Dynamics of genomic clones in breast cancer patient xenografts at single-cell resolution. Nature 518: $422-426$

Gao H, Korn JM, Ferretti S, Monahan JE, Wang Y, Singh M, Zhang C, Schnell C, Yang G, Zhang Y, et al. 2015. Highthroughput screening using patient-derived tumor xenografts to predict clinical trial drug response. Nat Med 21: 1318-1325.

Garnett MJ, Edelman EJ, Heidorn SJ, Greenman CD, Dastur A, Lau KW, Greninger P, Thompson IR, Luo X, Soares J, et al.
2012. Systematic identification of genomic markers of drug sensitivity in cancer cells. Nature 483: $570-575$

Harrison RK. 2016. Phase II and phase III failures: 2013-2015. Nat Rev Drug Discov 15: 817-818.

Hidalgo M, Amant F, Biankin AV, Budinská E, Byrne AT, Caldas C, Clarke RB, de Jong S, Jonkers J, Mælandsmo GM, et al. 2014. Patient-derived xenograft models: An emerging platform for translational cancer research. Cancer Discov 4: 998-1013.

Pereira B, Chin SF, Rueda OM, Vollan HK, Provenzano E, Bardwell HA, Pugh M, Jones L, Russell R, Sammut SJ, et al. 2016. The somatic mutation profiles of 2,433 breast cancers refines their genomic and transcriptomic landscapes. Nat Commun 7: 11479.

Perou CM, Sørlie T, Eisen MB, van de Rijn M, Jeffrey SS, Rees CA, Pollack JR, Ross DT, Johnsen H, Akslen LA, et al. 2000. Molecular portraits of human breast tumors. Nature 406: $747-752$.

Roth A, Khattra J, Yap D, Wan A, Laks E, Biele J, Ha G, Aparicio S, Bouchard-Côté A, Shah SP. 2014. PyClone: Statistical inference of clonal population structure in cancer. Nat Methods 11: 396-398.

Tentler JJ, Tan AC, Weekes CD, Jimeno A, Leong S, Pitts TM, Arcaroli JJ, Messersmith WA, Eckhardt SG. 2012. Patientderived tumour xenografts as models for oncology drug development. Nat Rev Clin Oncol 9: 338-350.

van de Wetering M, Francies HE, Francis JM, Bounova G, Iorio F, Pronk A, van Houdt W, van Gorp J, Taylor-Weiner A, Kester L, et al. 2015. Prospective derivation of a living organoid biobank of colorectal cancer patients. Cell 161: 933945. 


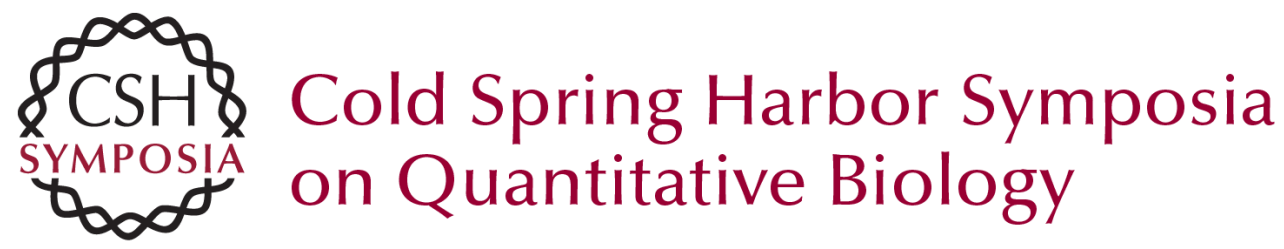

\section{Modeling Breast Cancer Intertumor and Intratumor Heterogeneity Using Xenografts}

Alejandra Bruna, Oscar M. Rueda and Carlos Caldas

Cold Spring Harb Symp Quant Biol 2016 81: 227-230 originally published online April 5, 2017 Access the most recent version at doi:10.1101/sqb.2016.81.031112

References This article cites 15 articles, 1 of which can be accessed free at: http://symposium.cshlp.org/content/81/227.full.html\#ref-list-1

Creative This article is distributed under the terms of the

Commons http://creativecommons.org/licenses/by-nc/4.0/, which permits reuse and License redistribution, except for commercial purposes, provided that the original author and source are credited.

Email Alerting Receive free email alerts when new articles cite this article - sign up in Service the box at the top right corner of the article or click here. 